\title{
Bronchialkarzinom-Früherkennung
}

\section{CT-Screening für Raucher?}

\author{
Regelmäßige Früherkennungs- \\ untersuchungen mit Niedrig- \\ dosis-CT senken bei starken \\ Rauchern die Lungenkrebs- \\ Sterblichkeit. So heißt es in \\ einer Pressemitteilung des \\ National Cancer Institute zu \\ einer noch nicht im Original \\ publizierten Studie. Die \\ Deutsche Gesellschaft für \\ Pneumologie und die \\ Deutsche Röntgengesellschaft \\ warnen aus diesem Anlass vor \\ einer unkritischen Anwendung \\ von CT-Untersuchungen.
}

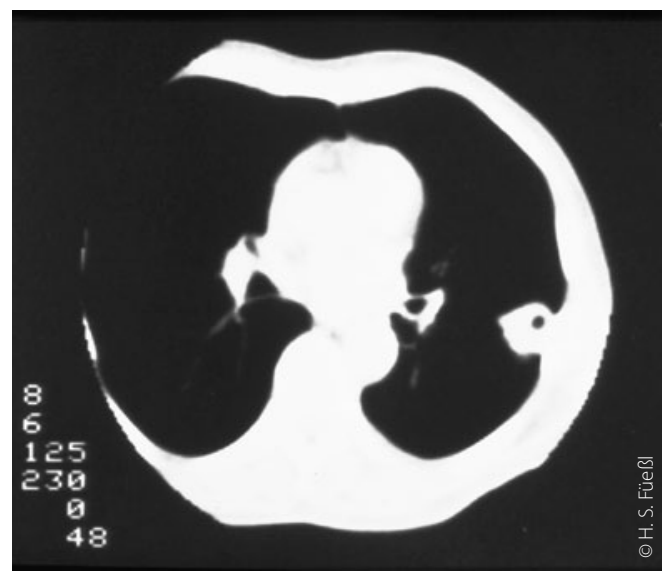

Wandständiges Bronchialkarzinom.
- Bei drei von vier Patienten ist ein Bronchialkarzinom bei seiner Entdeckung bereits nicht mehr operabel. Ihre Prognose ist entsprechend düster: Nach fünf Jahren leben nicht einmal mehr 5\% von ihnen, sagte Dr. David Felix Heigener, Krankenhaus Grosshansdorf. Jetzt gebe es erstmals Hinweise, dass ein Screening die Prognose von Hochrisikopatienten verbessern könne. Im National Lung Screening Trial senkten regelmäßige Spiral-CT-Untersuchungen die Lungenkrebssterblichkeit von starken Rauchern relativ um $20 \%$, so Heigener.

An der bisher nur als Pressemitteilung veröffentlichten Studie beteiligten sich knapp 54000 Personen zwischen 55 und 74 Jahren, die mindestens 30 Jahre lang täglich 20 Zigaretten geraucht hatten. Sie erhielten dreimal hintereinander im Jahresabstand entweder ein Niedrigdosis-CT oder eine Röntgenthorax-Aufnahme. In den folgenden fünf Jahren ereigneten sich in der CT-Gruppe 354 Todesfälle (1,338\%) und in der Gruppe mit konventionellem Röntgen 442 $(1,671 \%)$ Todesfälle.

\section{Worüber Sie Ihre Patienten aufklären sollten}

Die deutschen Fachgesellschaften für Pneumologie und für Radiologie haben auf die Veröffentlichung dieser Daten mit einer gemeinsamen Stellungnahme reagiert. Sie befürchten, dass Anfragen von Rauchern wegen CT-Untersuchungen zunehmen und wollen den unkritischen Einsatz der Computertomografie verhindern. Sie raten deswegen zu einer ausführlichen Aufklärung von $\mathrm{Pa}$ tienten mit den folgenden Botschaften. (1) Die Ergebnisse der Studie sind möglicherweise nicht übertragbar auf 50 Jährige, die „nur“ 20 Jahre gequalmt haben.

(2) Bei einem Screening würde es "Grenzwertbefunde hageln", so Heigener. Falsch positive Befunde können unnötige und mit einem Risiko belastete Operationen nach sich ziehen.

(3) Bei einem großen Teil der Patienten wird der Lungenkrebs nur früher diagnostiziert werden - ohne dass ihre Heilungschancen steigen.

4 Die regelmäßige Strahlenexposition geht selbst mit einem erhöhten Krebsrisiko einher.

5 Der beste Schutz vor Lungenkrebs ist immer noch der Verzicht aufs Rauchen! „Es kann nicht sein, dass wir den Patienten indirekt vermitteln ,raucht weiter! - mit dem CT sehen wir schon rechtzeitig, wenn etwas passiert", sagte Heigener.

BS =

- Quelle: Eröffnungspressekonferenz, DGP-Jahrestagung, Dresden, April 2011 